\title{
A NOTE ON INNER ACTIONS OF HOPF ALGEBRAS
}

\author{
STEFAAN CAENEPEEL
}

(Communicated by Maurice Auslander)

\begin{abstract}
Let $H$ be a commutative, cocommutative, and faithfully projective Hopf algebra over a commutative ring $R$. A twisted version of inner action of a Hopf algebra, called $H$-inner action is introduced, and it is shown that $H$ acts $H$-innerly on an $H$-Azumaya algebra, if $\operatorname{Pic}\left(H^{*}\right)$ is trivial.
\end{abstract}

\section{INTRODUCTION}

Let $H$ be a Hopf algebra over a commutative ring $R$, and suppose that $H$ acts on an $R$-algebra $A$. Sweedler [19] introduced the notion of inner action of $H$ on $A: H$ is said to be acting innerly on $A$ if there exists a convolution invertible $u \in \operatorname{Hom}_{R}(H, A)$ such that for all $h \in H, a \in A$, $h \rightarrow a=\Sigma_{(h)} u\left(h_{2}\right) a v\left(h_{(2)}\right)$, where $v$ is the convolution inverse of $u$. A natural question is the following: when is a Hopf algebra action inner? The case where $R$ is a field has been studied by several authors, notably [6,10,13,17]; a nice survey was recently published by Montgomery (cf. [16]). In the case of an arbitrary commutative ring, Beattie [4, 5] and Masuoka [15] recently obtained significant results.

Most cases studied in the literature reduce to the case where $A$ is a central simple algebra, or more generally, an Azumaya algebra. Under certain conditions on $R, H$, and $A$, one obtains that the action of $H$ on $A$ is inner; in view of the form of the formula stated above, it is not surprising that these results are called "Skolem-Noether-like" theorems. In this note we will show that, at least in the case where $H$ is cocommutative and faithfully projective, this type of result is not only "Skolem-Noether-like", but actually follows from the SkolemNoether Theorem. We will apply the following trick: if $H$ is cocommutative, then $H^{*}$ is a commutative $R$-algebra, and $A \otimes H^{*}$ is an Azumaya algebra, with groundring $H^{*}$, on which we will apply the classical Skolem-Noether Theorem.

Although Proposition 2.7 is a special case of results obtained in $[5,15]$, we have decided to include full details of our new proof. Actually this method of proof allows us to study Hopf algebra actions on $\mathrm{H}$-dimodule algebras. An

Received by the editors April 10, 1990 and, in revised form, September 11, 1990. 1980 Mathematics Subject Classification (1985 Revision). Primary 16A24, 16A16.

Key words and phrases. Azumaya algebra, Hopf algebra, dimodule algebra. 
$H$-dimodule algebra is an algebra furnished with a compatible $H$-module and $H$-comodule structure. For a definition and basic properties of $H$-modules, $H$-comodules, and $H$-dimodules, we refer to the literature (e.g. $[1,14,20]$ ). We will introduce a kind of "twisted" version of inner action, called $H$-inner action, and we will show that, if $A$ is an $H$-Azumaya algebra in the sense of Long [14], and if $\operatorname{Pic}\left(H^{*}\right)=1$, then $H$ acts $H$-innerly on $A$.

\section{Notations AND PRELIMINARY RESUltS}

Let $R$ be a commutative ring, and $H$ a faithfully projective Hopf algebra, that is, a Hopf algebra which is finitely generated, faithfully flat, and projective as an $R$-module. The structural maps of $H$ will be denoted by $\Delta_{H}$ (the diagonal), $\varepsilon_{H}$ (the counit), $m_{H}$ (the multiplication), $\eta_{H}$ (the unit), and $S_{H}$ (the antipode). The subscript $H$ will be omitted whenever no confusion is possible. We will use Sweedler's $\Sigma$-notation extensively; for example, we will write, for $h \in H: \Delta h=\Sigma_{(h)} h_{(1)} \otimes h_{(2)},(\Delta \otimes 1) \Delta h=(1 \otimes \Delta) \Delta h=\Sigma_{(h)} h_{(1)} \otimes h_{(2)} \otimes h_{(3)}$, etc. For more details, cf. [20].

The group of grouplike elements of $H$ will be denoted by $G(H)$. For the structural map $\psi: H \otimes M \rightarrow M$ of an $H$-module $M$, we will write $\psi(h \otimes m)=$ $h \rightarrow m$, and for the structural map $\chi: M \rightarrow M \otimes H$ of an $H$-comodule, we will use Sweedler's notation: $\chi(m)=\Sigma_{(m)} m_{(0)} \otimes m_{(1)}$. Given two $H$-dimodule algebras $A$ and $B$, we define the smash product $A \# B$ of $A$ and $B$ to be $A \otimes B$ as an $H$-dimodule, and with algebra structure defined by $(a \# b)(c \# d)=$ $\Sigma_{(b)} a\left(b_{(1)} \rightarrow c\right) \# b_{(0)} d$.

The $H$-opposite algebra $\bar{A}$ of an $H$-dimodule algebra $A$ is equal to $A$ as an $H$-dimodule, but with multiplication structure given by $\bar{a} \cdot \bar{b}=\overline{\Sigma_{(a)}\left(a_{(1)} \rightarrow b\right) a_{(0)}}$.

Suppose that $H$ is a commutative, cocommutative, and faithfully projective Hopf algebra. Long [14] showed that the maps

$$
F: A \# \bar{A} \rightarrow \operatorname{End}_{R}(A) \text { and } G: \bar{A} \# A \rightarrow \operatorname{End}_{R}(A)^{\text {opp }}
$$

defined by

$$
F(a \# \bar{b})(c)=\Sigma_{(b)} a\left(b_{(1)} \rightarrow c\right) b_{(0)} \quad \text { and } \quad G(\bar{a} \# b)(c)=\Sigma_{(c)}\left(c_{(1)} \rightarrow a\right) c_{(0)} b
$$

are homomorphisms of $H$-dimodule algebras. If $A$ is faithfully projective as an $R$-module, and if the maps $F$ and $G$ defined above are isomorphisms, then $A$ is called an $H$-Azumaya algebra (cf. $[2,14]$ for more details on $H$-Azumaya algebras).

The main purpose of this note is to study Hopf algebra actions on $H$ Azumaya algebras. At first glance, one would expect that an $H$-Azumaya algebra is nothing else then an Azumaya algebra which is also an $H$-dimodule algebra; this is not always true, since an $H$-Azumaya algebra is not necessarily $R$-central (in fact, it is left and right $H$-central, and that is a weaker notion). These noncentral $H$-Azumaya algebras are an interesting source of algebras on which a Hopf algebra does not act innerly. Nevertheless, $H$-Azumaya algebras satisfy properties that are similar to the classical properties of an Azumaya algebra. 
For example, they may be classified into a Brauer group that is usually called the Brauer-Long group.

Let $\mathbf{P D}(R, H)$ be the category of invertible $H$-dimodules and $H$-dimodule homomorphisms; then the Grothendieck group of this category may be viewed as a dimodule version of the Picard group:

$$
P D(R, H)=K_{0} \mathbf{P D}(R, H) .
$$

This invariant of $R$ and $H$ may be easily linked to $\operatorname{Pic}(R), H$ and $H^{*}$ :

1.1. Proposition [7, Proposition 1.6]. $P D(R, H) \cong \operatorname{Pic}(R) \times G(H) \times G\left(H^{*}\right)$.

Proof. For details, we refer to [7, Proposition 1.6]. We restrict this paper to giving a description of the isomorphism $\alpha$ : $\operatorname{Pic}(R) \times G(H) \times G\left(G^{*}\right) \rightarrow P D(R, H)$. For $\left([I], h, h^{*}\right) \in \operatorname{Pic}(R) \times G(H) \times G\left(H^{*}\right)$, we define $\alpha\left([I], h, h^{*}\right)=\left[I\left(h, h^{*}\right)\right]$, where $I\left(h, h^{*}\right)$ is equal to $I$ as an $R$-module, and with dimodule structure defined by $\chi(x)=x \otimes h$ for all $x \in I$, and $k \rightarrow x=h^{*}(k) x$ for all $k \in H$, $x \in I$.

For an Azumaya algebra over a ring with trivial Picard group, we have the Skolem-Noether theorem, that is, every automorphism of $A$ is inner. For an $H$-Azumaya algebra, this is not always true, and this is why we introduce the notions of $H$-inner and $H$-INNER automorphisms. As before, let $H$ be a faithfully projective, commutative, and cocommutative Hopf algebra, and let $A$ be an $H$-Azumaya algebra. Let $H$-Aut $(A)$ be the group of all $H$-dimodule $R$-algebra automorphisms of $A$. We call $f \in H$-Aut $(A) H$-INNER, if there exists an invertible $x \in A$ such that

(1) $f(a)=x a x^{-1}$, for all $a \in A$;

(2) $\chi(x)=x \otimes 1$

(3) $h \rightarrow x=\varepsilon(h) x$, for all $h \in H$.

The subgroup of $H$-Aut $(A)$ consisting of $H$-INNER automorphisms of $A$ will be denoted by $H$-INN $(A) . \quad f \in H$-Aut $(A)$ will be called $H$-inner if there exists an invertible $x \in A$, and $h^{*} \in G\left(H^{*}\right)$ such that for all $a \in A: f(a)=$ $\Sigma_{(a)} h^{*}\left(a_{(1)}\right) x a_{(0)} x^{-1}$. We have the following generalization of the RosenbergZelinsky exact sequence:

1.2. Proposition (Dimodule version of the Rosenberg-Zelinsky sequence). For an H-Azumaya algebra $A$, we have the following short exact sequence:

$$
1 \rightarrow H \text {-INN }(A) \rightarrow H \text {-Aut }(A) \stackrel{\Phi}{\longrightarrow} P D(R, H)
$$

where $\Phi(f)=I_{f}$, with

$$
I_{f}=\left\{x \in A: \Sigma_{(a)}\left(a_{(1)} \rightarrow x\right) a_{(0)}=f(a) x, \text { for all } a \in A\right\} .
$$

Proof. We leave it as an exercise to the reader to modify the proof of the exactness of the classical Rosenberg-Zelinsky sequence, as presented for example in [12, IV.1.2]. Let us remark that instead of using the classical Morita equivalence, one has to use the $H$-dimodule version of the Morita equivalences as it is discussed by Beattie in $[2,3.6]$. 
1.3. Corollary (Generalized Skolem-Noether Theorem). If $\operatorname{Pic}(R)=1$, then every $f \in H$-Aut $(A)$ in $H$-inner.

Proof. Since $\left[I_{f}\right]=1$ in $\operatorname{Pic}(R), I_{f}=R x$ for some invertible $x \in A$. Let $\left[I_{f}\right]=\left([R], h, h^{*}\right)$ in $P D(R, H) \cong \operatorname{Pic}(R) \times G(H) \times G\left(H^{*}\right)$ (cf. Proposition 1.3). Then $k \rightarrow x=h^{*}(k) x$ for all $k \in H$, and the result follows from the preceding theorem.

\section{Hopf Algebra actions on $H$-AZUmaya algebras}

2.1. The Hopf algebra $\operatorname{Hom}_{R}(H, K)$. Let $H, K$ be faithfully projective Hopf algebras, and suppose that $H$ is cocommutative. $\underline{H}^{*}$ will be $H^{*}$, viewed as a commutative $R$-algebra (that is, we forget the coalgebra structure of $H^{*}$ ).

Let $\mathscr{H}=\operatorname{Hom}_{R}(H, K)$. An $H^{*}$-Hopf algebra structure may be defined on $\mathscr{H}$ as follows: the multiplication is given by the convolution, and the unit map $\eta_{\mathscr{H}}: \underline{H}^{*} \rightarrow \mathscr{H}$ is given by $\eta_{\mathscr{H}}\left(h^{*}\right)=\eta_{K} \circ h^{*}$. The diagonal map

$$
\Delta_{\mathscr{H}}: \mathscr{H} \rightarrow \mathscr{H} \otimes_{\underline{H}^{*}} \mathscr{H} \cong \operatorname{Hom}_{R}(H, K \otimes K)
$$

is defined by

$$
\Delta_{\mathscr{L}}(\mu)=\Delta_{K} \circ \mu
$$

for all $\mu \in \mathscr{H}$. The counit $\varepsilon_{\mathscr{H}}: \mathscr{H} \rightarrow \underline{H}^{*}$ is given by $\varepsilon_{\mathscr{C}}(\mu)=\varepsilon_{K} \circ \mu$, and the antipode $S_{\mathscr{H}}: \mathscr{H} \rightarrow \mathscr{H}$ by $S_{\mathscr{H}}(\mu)=S_{K} \circ \mu$.

2.2. Lemma. We have an isomorphism of $\underline{H}^{*}$-Hopf algebras $\alpha: \underline{H}^{*} \otimes K \rightarrow \mathscr{H}$ given by

$$
\alpha\left(h^{*} \otimes k\right)(h)=h^{*}(h) k,
$$

for all $h^{*} \in H^{*}, k \in K$, and $h \in H$.

Proof. Using the hom-tensor relations, we obtain that $\underline{H}^{*} \otimes K=\operatorname{Hom}_{R}(H, R) \otimes$ $K \cong \operatorname{Hom}_{R}(H, K)=\mathscr{H}$ as $R$-modules, and the isomorphism is given by $\alpha$. It is straightforward to check that $\alpha$ preserves the structure maps.

2.3. Lemma. $G(\mathscr{H})=\{\mu \in \mathscr{H}: \mu$ is a coalgebra homomorphism $\}$.

Proof. $\mu$ is grouplike if and only if $\Delta_{\mathscr{H}}(\mu)=\mu \otimes \mu$ and $\varepsilon_{\mathscr{H}}(\mu)=\varepsilon_{H}$, the unit in $\underline{H}^{*}$. The latter condition is equivalent to $\varepsilon_{K} \circ \mu=\varepsilon_{H}$. We have seen above that

$$
\left(\Delta_{\mathscr{H}}(\mu)\right)(h)=\Delta_{K}(\mu(h))=\Sigma_{\mu(h)} \mu(h)_{(0)} \otimes \mu(h)_{(1)}
$$

$\mu \rightarrow \mu \otimes \mu$ may be viewed as a map

$$
\begin{aligned}
\mathscr{H} & =\operatorname{Hom}_{R}(H, K) \cong \underline{H}^{*} \otimes K \rightarrow\left(\underline{H}^{*} \otimes K\right) \otimes_{\underline{H}^{*}}\left(\underline{H}^{*} \otimes K\right) \\
& \cong \underline{H}^{*} \otimes K \otimes K \cong \operatorname{Hom}_{R}(H, K \otimes K) .
\end{aligned}
$$

One may easily establish that $\mu \otimes \mu$ is given by $(\mu \otimes \mu)(h)=\Sigma_{(h)} \mu\left(h_{(0)}\right) \otimes \mu\left(h_{(1)}\right)$, for all $h \in H$ and the result follows. 
2.4. Lemma. $\mathscr{H}^{*}=\operatorname{Hom}_{R}(H, K)^{*} \cong \operatorname{Hom}_{R}\left(H, K^{*}\right)$, where * means the dual with respect to the groundring $\underline{H}^{*}$.

Proof. The result follows quite easily:

$$
\mathscr{H}^{*}=\operatorname{Hom}_{R}(H, K)^{*} \cong\left(\underline{H}^{*} \otimes K\right)^{*} \cong \underline{H}^{*} \otimes K^{*} \cong \operatorname{Hom}_{R}\left(H, K^{*}\right) .
$$

For later use, we give an explicit description of the action of $\mathscr{H}^{2}$ on $\mathscr{H}$. The action of $\underline{H}^{*} \otimes K^{*}$ on $\underline{H}^{*} \otimes K$ is given by

$$
\left\langle h^{*} \otimes k^{*}, g^{*} \otimes k\right\rangle=k^{*}(k) h^{*} * g^{*},
$$

for all $h^{*}, g^{*} \in H^{*}, k \in K, k^{*} \in K^{*}$. Translating this into an action of $\mathscr{H}^{*}$ on $\mathscr{H}$, we obtain, for $\mu^{*} \in \mathscr{H}^{*}, \mu \in \mathscr{H}:\left\langle\mu^{*}, \mu\right\rangle \in \underline{H}^{*}$ is defined by

$$
\left\langle\mu^{*}, \mu\right\rangle(h)=\Sigma_{(h)}\left\langle\mu^{*}\left(h_{(1)}\right), \mu\left(h_{(2)}\right)\right\rangle
$$

for all $h \in H$.

Now, suppose that $A$ is a $K$-module algebra; then $\mathscr{A}=\operatorname{Hom}_{R}(H, A)$ is an $\mathscr{H}$-module algebra. The algebra structure on $\mathscr{A}$ is given by the convolution, and the $\mathscr{H}$-action is given by

$$
\left.(\mu \rightarrow u)(h)=\Sigma_{(h)} \mu\left(h_{(1)}\right) \rightarrow u\left(h_{(2)}\right)\right)
$$

for all $h \in H, \mu \in \mathscr{H}, u \in \mathscr{A}$.

The proof of the following lemma is similar to the proof of Lemma 2.2.

2.5. Lemma. $\underline{H}^{*} \otimes A \cong \mathscr{A}$ as $\mathscr{H}$-module algebras.

Next, suppose that $H=K$, and define $F, G: \mathscr{A} \rightarrow \mathscr{A}$ by

$$
F(f)(h)=\Sigma_{(h)}\left(h_{(1)} \rightarrow f\left(h_{(2)}\right)\right) \quad \text { and } \quad G(f)(h)=\Sigma_{(h)}\left(\mathbf{S}\left(h_{(1)}\right) \rightarrow f\left(h_{(2)}\right)\right),
$$

for all $f \in \mathscr{A}, h \in H$.

2.6. Lemma. $F, G \in \mathscr{H}-\operatorname{Aut}(\mathscr{A})$, and $F$ and $G$ are each other's algebra automorphism inverses.

Proof. First, let us show that $F$ preserves the convolution. For all $f, g \in \mathscr{A}$ and $h \in H$ we have that

$$
\begin{aligned}
F(f * g)(h) & =\Sigma_{(h)} h_{(1)} \rightarrow\left(f\left(h_{(2)}\right) g\left(h_{(3)}\right)\right) \\
& =\Sigma_{(h)}\left(h_{(1)} \rightarrow f\left(h_{(2)}\right)\right)\left(h_{(3)} \rightarrow g\left(h_{(4)}\right)\right) \\
& =(F(f) * F(g))(h)
\end{aligned}
$$

If $h^{*} \in \underline{H}^{*}$, then we have for all $h \in H$ :

$$
\begin{aligned}
F\left(h^{*}\right)(h) & =\Sigma_{(h)} h_{(1)} \rightarrow h^{*}\left(h_{(2)}\right) \\
& =\Sigma_{(h)} \varepsilon\left(h_{(1)}\right) h^{*}\left(h_{(2)}\right) \\
& =h^{*}(h),
\end{aligned}
$$

so $F\left(h^{*}\right)=h^{*}$. 
Finally, it is straightforward to show that $G \circ F=F \circ G=\mathrm{Id}$.

We have an embedding $i: A \rightarrow \underline{H}^{*} \otimes A \cong \mathscr{A}$, given by $i(a)(h)=\varepsilon(h) a$ for all $a \in A, h \in H$. Similarly, we have an embedding $i: H \rightarrow \mathscr{H}$.

Also observe that $F(i(a))(h)=\Sigma_{(h)} h_{(1)} \rightarrow \varepsilon\left(h_{(2)}\right) a=h \rightarrow a$ for all $h \in H$, $a \in A$.

As an application, let us give an elementary proof of the following (cf. [5, 15] for a different approach):

2.7. Proposition. Let $H$ be a cocommutative faithfully projective Hopf algebra, and suppose that $\operatorname{Pic}\left(\underline{H}^{*}\right)=1$. Then $H$ acts innerly on any $H$-module Azumaya algebra $A$.

Proof. Take $H=K$ in the above arguments. $\underline{H}^{*} \otimes A=\mathscr{A}$ is an $\underline{H}^{*}$-module Azumaya algebra, and therefore, the automorphism $F$ defined above is inner, by the (classical!) Skolem-Noether Theorem. So there exists $u \in \operatorname{Hom}_{R}(H, A)$, with convolution inverse $v$, say, such that $F(f)=u * f * v$, or $F(f)(h)=$ $\Sigma_{(h)} u\left(h_{(1)}\right) f\left(h_{(2)}\right) v\left(h_{(3)}\right)$ for all $f \in \mathscr{A}, h \in H$. Applying this formula to $f=i(a)$, we obtain:

$$
h \rightarrow a=F(i(a))(h)=\Sigma_{(h)} u\left(h_{(1)}\right) \varepsilon\left(h_{(2)}\right) a v\left(h_{(3)}\right)=\Sigma_{(h)} u\left(h_{(1)}\right) a v\left(h_{(2)}\right),
$$

so $H$ acts innerly on $A$.

The argument used in the proof of Proposition 2.7 may be generalized in order to study the action of a Hopf algebra $H$ on an $H$-Azumaya algebra. From now on, suppose that $H=K$ is a commutative, cocommutative faithfully projective Hopf algebra. We already know that $\mathscr{A}=\operatorname{Hom}_{R}(H, A)$ is an $\mathscr{H}$ module algebra. Now $\mathscr{A}$ has the structure of an $\mathscr{H}$-dimodule algebra if we define $\chi_{\mathscr{A}}: \mathscr{A} \rightarrow \mathscr{A} \otimes_{\mathscr{H}^{*}} \mathscr{H}$ by $\chi_{\mathscr{A}}(u)=\chi_{A} \circ u$. The isomorphism discussed in Lemma 2.5 is then an isomorphism of $\mathscr{H}$-dimodule algebras, and $\mathscr{A}$ is an $\mathscr{H}$-Azumaya algebra.

2.8. Theorem. Let $A$ be an $H$-Azumaya algebra. If $\operatorname{Pic}\left(H^{*}\right)=1$, then $H$ acts $H$-innerly on $A$, that is, there exists a convolution invertible $u \in \operatorname{Hom}_{R}(H, A)$, with convolution inverse $v$, and $\mu^{*} \in \operatorname{Hom}_{\text {bialg }}\left(H, H^{*}\right)$ such that for all $h \in H$, $a \in A$ :

$$
h \rightarrow a=\Sigma_{(h),(a)} \mu^{*}\left(h_{(1)}\right)\left(a_{(1)}\right) u\left(h_{(2)}\right) a_{(0)} v\left(h_{(3)}\right) .
$$

Proof. With notations as above, $\mathscr{A}$ is an $\mathscr{H}$-Azumaya algebra. We apply Proposition 1.2 to the automorphism $F$. Then $I_{F} \cong \underline{H}^{*}\left(\mu, \mu^{*}\right)$ with $\mu \in$ $G(\mathscr{H}), \mu^{*} \in G\left(\mathscr{H}^{*}\right)$. Let $I_{F}$ be generated $u \in \mathscr{A}=\operatorname{Hom}_{R}(H, A)$. Then $u$ is convolution invertible, and, for all $\lambda \in \mathscr{H}, \lambda \rightarrow u=\left\langle\mu^{*}, \lambda\right\rangle * u$ (cf. Proposition 1.1). For all $f \in \mathscr{A}$ we have that

$$
F(f) * u=\Sigma_{(f)}\left(f_{(1)} \rightarrow u\right) * f_{(0)},
$$

and therefore,

$$
F(f)=\Sigma_{(f)}\left(f_{(1)} \rightarrow u\right) * f_{(0)} * v
$$


Let $f=i(a)$ with $a \in A$. Then for all $h \in H$,

$$
\chi_{\mathscr{A}}(i(a))(h)=\chi_{A}(\varepsilon(h)(a))=\Sigma_{(a)} \varepsilon(h) a_{(0)} \otimes a_{(1)},
$$

so

$$
\chi_{\mathscr{A}}(i(a))=\Sigma_{(a)} i\left(a_{(0)}\right) \otimes i\left(a_{(1)}\right) \in \mathscr{A} \otimes_{\underline{H}^{*}} \mathscr{H} .
$$

Now $i(a) \rightarrow u=\left\langle\mu^{*}, i(a)\right\rangle * u$, and for all $h \in H$,

$$
\begin{aligned}
\left\langle\mu^{*}, i(a)\right\rangle(h) & =\Sigma_{(h)} \mu^{*}\left(h_{(1)}\right)\left(i(a)\left(h_{(2)}\right)\right) \\
& =\Sigma_{(h)} \mu^{*}\left(h_{(1)}\right)\left(\varepsilon\left(h_{(2)}\right) a\right) \\
& =\mu^{*}(h)(a) .
\end{aligned}
$$

Therefore,

$$
F(i(a))=\Sigma_{(a)} \mu^{*}(\cdot)\left(a_{(1)}\right) * u * i\left(a_{(0)}\right) * v,
$$

and for all $h \in H$,

$$
F(i(a))(h)=h \rightarrow a=\Sigma_{(a),(h)} \mu^{*}\left(h_{(1)}\right)\left(a_{(1)}\right) u\left(h_{(2)}\right) a_{(0)} v\left(h_{(3)}\right) .
$$

It follows from Lemma 2.3 that $\mu^{*}$ is a coalgebra homomorphism, so the only thing that remains to be shown is that $\mu^{*}$ is an algebra homomorphism.

Let $\rho$ be the composition of the maps

$$
\begin{aligned}
\rho: \operatorname{Hom}_{R}(H \otimes H, H)-\operatorname{Aut}\left(\operatorname{Hom}_{R}(H \otimes H, A)\right) \\
\quad \stackrel{\Phi}{\rightarrow} P D\left((\underline{H \otimes H})^{*}, \operatorname{Hom}_{R}(H \otimes H, H)\right) \\
\quad \rightarrow G\left(\operatorname{Hom}_{R}\left(H \otimes H, H^{*}\right)\right) .
\end{aligned}
$$

Consider the following automorphisms $F_{0}, F_{1}, F_{2}$ of $\operatorname{Hom}_{R}(H \otimes H, A)$ :

$$
\begin{aligned}
& F_{0}(f)(h \otimes k)=\Sigma_{(k)} k_{(1)} \rightarrow f\left(h \otimes k_{(2)}\right), \\
& F_{1}(f)(h \otimes k)=\Sigma_{(h),(k)} h_{(1)} k_{(1)} \rightarrow f\left(h_{(2)} \otimes k_{(2)}\right), \\
& F_{2}(f)(h \otimes k)=\Sigma_{(h)} h_{(1)} \rightarrow f\left(h_{(2)} \otimes k\right) .
\end{aligned}
$$

If $u \in I_{F}$, then $\varepsilon \otimes u \in I_{F_{0}}, u \circ m_{H} \in I_{F_{1}}, u \otimes \varepsilon \in I_{F_{2}}$, and therefore,

$$
\rho\left(F_{0}\right)=\varepsilon \otimes \mu^{*} ; \quad \rho\left(F_{1}\right)=\mu^{*} \circ m_{H} ; \quad \rho\left(F_{2}\right)=\mu^{*} \otimes \varepsilon .
$$

Now $F_{2} F_{1}^{-1} F_{0}$ is the identity, so

$$
\rho\left(F_{2}\right) \rho\left(F_{1}\right)^{-1} \rho\left(F_{0}\right)=\varepsilon .
$$

It follows that for all $h, k \in H$,

$$
\left(\mu^{*} \circ m_{H}\right)(h \otimes k)=\left(\left(\varepsilon \otimes \mu^{*}\right) *\left(\mu^{*} \otimes \varepsilon\right)\right)(h \otimes k),
$$

and

$$
\mu^{*}(h k)=\mu^{*}(h) * \mu^{*}(k)
$$

\subsection{Examples.}

(2.9.1) Let $G$ be a finite abelian group, and let $H=R G$. Then $\operatorname{Hom}_{\text {bialg }}\left(H, H^{*}\right) \cong \operatorname{Hom}\left(G(H), G\left(H^{*}\right)\right)=\operatorname{Hom}\left(G, G^{*}\right)$, 
and for an $R G$-Azumaya algebra $A$,

$$
\begin{gathered}
\operatorname{Hom}_{R}(H, A) \cong \operatorname{Map}(G, A) \\
\mathbb{G}_{m}\left(\operatorname{Hom}_{R}(H, A)\right) \cong \operatorname{Map}\left(G, \mathbb{G}_{m}(A)\right) .
\end{gathered}
$$

It is also clear that the convoiution on $\operatorname{Hom}_{R}(H, A)$ corresponds to pointwise multiplication on $\operatorname{Map}(G, A)$. Suppose that $\operatorname{Pic}(R)=1$; then $\operatorname{Pic}\left((R G)^{*}\right)$ $=\operatorname{Pic}(G R)$ is the direct sum of $|G|$ copies of $\operatorname{Pic}(R)$, so $\operatorname{Pic}\left((G R)^{*}\right)$ is trivial. Theorem 2.8 implies that there exists $\mu^{*} \in \operatorname{Hom}\left(G, G^{*}\right)$ and for all $\sigma \in G$, $u_{\sigma} \in \mathbb{G}_{m}(A)$ such that for all homogeneous $a \in A: \sigma \cdot a=\mu^{*}(\sigma)(\alpha) u_{\sigma} a u_{\sigma}^{-1}$, where $\alpha$ is the grade of $a$; this result has been exploited by the author and M. Beattie in [8].

(2.9.2) Let us give a more specific example: let $R=\mathbb{Z}[\sqrt{2}]$ and $H=$ $R[x] /\left(x^{2}-\sqrt{2} x\right)$, with coalgebra structure defined by $\Delta x=x \otimes 1+1 \otimes x-$ $\sqrt{2} x \otimes x, \varepsilon x=0$, and $S=$ Id. For a more detailed discussion of this Hopf algebra, we refer to $[9,11]$.

The map $f: H \rightarrow H^{*}$, defined by $f(1)=\varepsilon$ and $f(x)=y$, where $y(x)=$ -1 , is an isomorphism of Hopf algebras. Let $A$ be equal to $H$ as an $R$-algebra, and define an $H$-module and $H$-comodule structure on $A$ by $\chi(h)=\Delta h$, for all $h \in H, x \rightarrow 1=0$, and $x \rightarrow x=\sqrt{2} x-1$. Then $A$ is an $H$-Azumaya algebra (cf. [7, Proposition 4.3]); a lengthy, but straightforward computation shows that the formula Theorem 2.8 holds if we take $u=v=$ Id and $\mu^{*}: H \rightarrow H^{*}$ equal to the map $f$ defined above.

\section{REFERENCES}

1. E. Abe, Hopf algebras, Cambridge Univ. Press, Cambridge, 1977.

2. M. Beattie, Brauer groups of $H$-module and $H$-dimodule algebras, thesis, Queens Univ., Kingston, Ontario, 1976.

3. __ Automorphisms of G-Azumaya algebras, Canad. J. Math. 37 (1985), 1047-1058. Correction to Automorphisms of G-Azumaya algebras, preprint.

4. __ Inner gradings and Galois extensions with normal basis, Proc. Amer. Math. Soc. 107 (1989), 881-886.

5. M. Beattie and K.-H. Ulbrich, A Skolem-Noether theorem for Hopf algebra actions, Comm. Algebra (to appear).

6. R. J. Blattner, M. Cohen, and S. Montgomery, Crossed products and inner actions of Hopf Algebras, Trans. Amer. Math. Soc. 298 (1986), 671-711.

7. S. Caenepeel, Computing the Brauer-Long group of a Hopf algebra I: the cohomological theory, Israel J. Algebra (to appear).

8. S. Caenepeel and M. Beattie, A cohomological approach to the Brauer-Long group and the groups of Galois extensions and strongly graded rings, Trans. Amer. Math. Soc. (to appear).

9. L. N. Childs, Representing classes in the Brauer group of quadratic number rings as smash products, Pacific J. Math. 129 (1987).

10. M. Cohen, Smash products, inner action and quotient rings, Pacific J. Math. 125 (1986), 45-66.

11. S. Hurley, Galois objects with normal bases for free Hopf algebras of prime degree, J. Algebra 109 (1987), 292-318. 
12. M. A. Knus and M. Ojanguren, Théorie de la descente et algèbres d'Azumaya, Lecture Notes in Math., vol. 389, Springer-Verlag, Berlin, 1974.

13. M. Koppinen, A Skolem-Noether Theorem for Hopf algebra measurings, preprint.

14. F. Long, The Brauer group of dimodule algebras, J. Algebra 30 (1974), 559-601.

15. A Masuoka, Coalgebra actions on Azumaya algebras, Tsukuba J. Math. (to appear).

16. S. Montgomery, Inner actions of Hopf algebras, Ring Theory, 1989, The Weizmann Science Press of Israel, Jerusalem, 1989, pp. 141-149.

17. J. Osterburg and D. Quinn, A Skolem-Noether theorem for group graded rings, J. Algebra 113 (1988), 483-490; Addendum to A Skolem-Noether theorem for group graded rings, J. Algebra 120 (1989), 414-415.

18. A. Rosenberg and D. Zelinsky, Automorphisms of separable algebras, Pacific J. Math. 11 (1961), 1107-1117.

19. M. E. Sweedler, Cohomology of algebras over Hopf algebras, Trans. Amer. Math. Soc. 133 (1968), 205-239.

20. __, Hopf algebras, Benjamin, New York, 1969.

Faculty of Applied Sciences, Free University of Brussels, VUB, PleinlaAn 2, B-1050 BRUSSELS, BeLgium 